\title{
X-RAY IRRADIATED ACCRETION DISK AND BIMODAL STATES
}

\author{
R. Hoshi ${ }^{1}$ and H. Inoue ${ }^{2}$ \\ 1 Department of Physies, Rikkyo University, \\ Nishi-Ikebukuro, Tokyo 171 \\ 2 Institute of Space and Astronautical Science, \\ 4-6-1 Komaba, Meguro-ku, Tokyo 153
}

It is well known that black hole candidates, Cyg $\mathrm{X}-1$ and GX 339-4, have distinct high and low states, known as bimodal states. Detailed spectroscopic studies of these $X$-ray sources have revealed high and low states corresponding to optically thick and thin states of the surrounding accretion disks.

We have found that bimodal states are the natural consequence of the irradiation of the outer accretion disk by $\mathrm{X}$-rays emanating from the vicinity of the accreting black hole.

It has been found by Hayakawa that the outer accretion disk is heated by Compton scattering of $\mathrm{X}$-rays emerging from the vicinity of the accreting black hole. As a result, even if the outer disk is initially optically thick (geometrically thin), Compton heated hot gas continuously evapolates from the low density surface layer of the optically thick disk and finally forms an optically thin hot outer disk. This optically thin disk is maintained by $X$-ray heating (external source) if the accretion rate is larger than a certain critical value Mcr. If the accretion rate is lower than Mcr, the disk is maintained by viscous heating (internal source).

For the former case, as we proceed inward, the disk changes its structure to an optically thick-geometrically thin state, since cooling due mainly to free-free emission dominates over $\mathrm{X}$-ray heating. This optically thick disk extends down to the radius of the innermost stable circular orbit. Soft $\mathrm{X}$-rays are exclusively emitted from the optically thick disk surface in accord with the soft $X$-ray excess observed in the high states of Cyg X-1 and GX 339-4.

When the accretion rate is decreased below Mcr, the disk is maintained by viscous heating. In this case, no cooling process outweighs viscous heating. The disk remains optically thin down to the vicinity of the black hole, which corresponds to the low states characterized by a power law spectrum in the X-ray range. Thus, a high to low state transition occurs when the accretion rate is decreased below Micr. In order to change to a high state, an increase in the accretion rate of more than 3 Mer is required. This hysteretic behavior explains why black hole candidates remain in one state for weeks or months before changing to the other state. 